\title{
ECG Alterations Precedes Cardiac Hypertrophy in Rat Model of Diabetes
}

\author{
Charu Gupta ${ }^{1}$, Swati Omanwar ${ }^{1 *}$, Parvesh Bubber ${ }^{1}$ and Bano Saidullah ${ }^{1}$ \\ School of Sciences, Indira Gandhi National Open University (IGNOU), New Delhi
}

Submission: December 07, 2017; Published: March 20, 2018

*Corresponding author: Swati Omanwar, School of Sciences, Indira Gandhi National Open University (IGNOU), Maidan Garhi-110068, New Delhi, Tel: 91-01129572818; Email: swatiomanwar@gmail.com

\begin{abstract}
Diabetes induced Cardiovascular Diseases (CVD) are a major reason for mortality in diabetic patients. ECG a noninvasive tool is used for early detection of CVD. Therefore, the present study was designed to evaluate the effect of diabetes on cardiac electrical conduction in Wistar rats. Rat model of diabetes was developed by feeding Wistar rats high fat diet (HFD) for three weeks along with Streptozotocin (STZ) administration (25 mg/kg, i.p.) on second week. Rats fed HFD and injected STZ showed hyperglycemia, a significant alteration in ECG profile along with cardiac injury but devoid of cardiac hypertrophy. Our results indicate that in diabetes cardiac electrical conduction defects set in first before the structural changes appear in the heart. These subtle cardiac electrical changes may be the precipitating factor for the cardiovascular diseases in diabetes.

Keywords: Diabetes; Electrocardiography; Streptozotocin; High fat diet; Hypertrophy.
\end{abstract}

\section{Introduction}

Diabetes Mellitus (DM) is the most persistent disease and is considered a Cardiovascular Disease (CVD) risk equivalent [1,2]. CVD accounts for the majority of deaths in people with type 2 diabetes mellitus (T2DM). T2DM has been associated with elevated risk for myocardial infarction, heart failure, and sudden cardiac death. Hyperglycemia is associated with left ventricular hypertrophy in T2DM [2]. In diabetes, myocardial damage and fibrosis are results of pathological cardiac hypertrophy [3-5] which leads to defects in the filling and contractility of the heart. Subsequently, cardiac dysfunction presents as decreased activity tolerance, ejection fraction, cardiac output and heart failure [6]. Changes in QRS, PR, QT intervals, P-wave morphology, and heart-rate variability have been associated with diabetes [7-14]. An abnormal Electrocardiogram (ECG) is a marker of high risk associated with cardiac mortality and morbidity [15]. The ECG offers a quick, non-invasive technique for the early detection of CVDs. Hence, a better perception of how the electrical cardiac conduction is affected by diabetes is much needed.

Several animal models of T2DM have been developed, though not a single animal model is exactly same as human T2DM induced cardiac disorders [16]. Though a number of genetic diabetic models are used to study a particular pathway, such models are not useful to study multiple pathways involved in the development of cardiac dysfunction in diabetes holistically [17-19]. Recently a new rat model was proposed by
Mansor et al. [20] which induces T2DM by using High Fat Diet (HFD) followed by a low dose of the Streptozotocin (STZ) [20]. At a dose of $25 \mathrm{mg} / \mathrm{kg} \mathrm{STZ}$ in combination with HFD feeding for three weeks caused mild hyperglycemia, hypercholesterolemia and exhibited cardiac metabolic changes that mirror the decrease in glucose metabolism in diabetic patients. Though, at this dose cardiac metabolic changes were observed, no change in heart: body weight ratio was observed indicating no structural changes [20]. The present study was done to evaluate whether cardiac electrical conduction alteration set in before, cardiac structural changes occur in diabetes.

\section{Materials and Methods}

Wistar rats (250-300g) were obtained from the animal house of Indira Gandhi National Open University (IGNOU), Delhi. The care and use of laboratory animals were in accordance with the recommendations by National Accreditation Board of Testing and calibration Laboratories (NABL). All experimental protocols were approved by the Institutional Animal Ethical Committee, IGNOU, New Delhi, India, and experiments were performed according to the guidelines of the Committee for control and supervision of Experiments on Animals (CPCSEA), Government of India. During the experimental study period rats were housed at constant room temperature, humidity, and light cycle (12: 12 $\mathrm{h}$ light-dark), with free access to tap water and were fed with commercial standard chow ad libitum. 


\section{Grouping of Animals}

Twenty rats were divided into two groups each having 10 rats.

1. Control Group (healthy control): Wistar rats were fed with normal pellet diet for 3 weeks and were given a single intraperitoneal injection of citrate buffer (pH-4.5, $0.1 \mathrm{M}$, $0.5 \mathrm{ml}$ ) on 2 nd week of overright facing.

2. HFD+STZ (3 weeks) group: Rats were fed high-fat diet for 3 weeks, and were given a single intraperitoneal injection of STZ [(25 mg/kg) in citrate buffer, on 2nd week after overnight fasting.

All experiments were done one week after the intraperitoneal injection of citrate buffer and STZ were given in control and experimental animals respectively. Body weights of all the experimental animals were monitored throughout the study.

\section{Fasting blood glucose (FBG)}

Blood glucose levels were measured from blood collected from rat tail using Accu-Check Active meter (Roche Diagnostics) before and at the end of the study i.e. 3 weeks.

\section{Electrocardiography (ECG)}

After the end of experimental period i.e. 3 weeks all the experimental animals were anesthetized with urethane $(1 \mathrm{~g} / \mathrm{Kg})$

Table 1: Body Weight Gain and LVW/WHW ratio. and ECG leads were connected to the dermal layer of both front paws and hind legs of animals from both the groups to Power lab data acquisition system (Chart v.8.1.8, AD Instruments, Australia) for the recording of ECG [21].

\section{Assessment of Cardiac injury}

The release of myocardial enzyme CK-MB into the circulation was estimated by using the commercially available enzymatic kit (Reckon India Pvt. Ltd.).

\section{Assessment of left ventricular hypertrophy:}

After the end of experimental period heart from rats of both groups were excised, and weight of the whole heart and left ventricle were measured. The ratio of left ventricle to whole heart weight (LVW/WHW) was calculated to assess left ventricular hypertrophy.

\section{Statistical Analysis}

The results are presented as mean \pm S.E.M. All data were analyzed by paired t-test. P value of less than 0.05 was considered as statistically significant.

\section{Results}

No significant change in body weight gain was observed in animals of both groups (Table 1 ).

\begin{tabular}{|c|c|c|}
\hline Group & Body Weight Gain (grams) & LVW/WHW (mg/gram) \\
\hline Control & $18.14 \pm 4.27$ & $38.92 \pm 0.99$ \\
\hline HFD+STZ (3 weeks) & $21.30 \pm 2.23$ & $40.13 \pm 0.75$ \\
\hline
\end{tabular}

Results represent mean \pm SEM of ten animals per group.

Estimation of Fasting blood glucose and assessment of Cardiac injury and cardiac hypertrophy

A marked elevation $(\mathrm{P}<0.005)$ in fasting blood glucose (Figure 1a) and serum cardiac injury marker i.e. CK-MB (Figure 1b) were observed in HFD+STZ (3 weeks) group in comparison to normal control group signifying that HFD+STZ causes hyperglycemia and myocardial damage. There was no change in $\mathrm{LVW} / \mathrm{HW}$ ratio in HFD+STZ (3 weeks) animals in comparison to the control rats (Table 1 ).

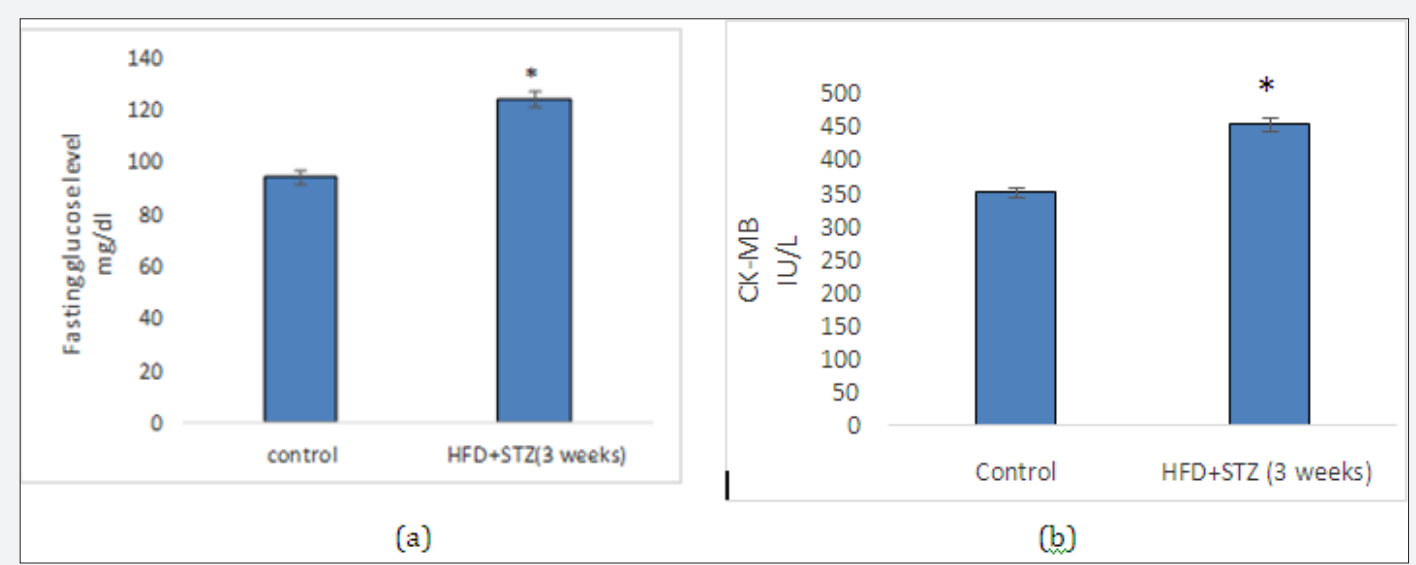

Figure 1: a) Fasting blood glucose and b) Serum CK-MB levels. Results are expressed as mean \pm SEM of ten animals per group. Result obtained are significantly different from control group $\left({ }^{*} p<0.05\right)$. 


\section{ECG parameters}

$\mathbf{P}$ amplitude and $\mathbf{P}$ wave duration : Significant $\left({ }^{*} \mathrm{p}<0.05\right)$ increase in $\mathrm{P}$ wave amplitude and $\mathrm{P}$ wave duration, which

Table 2: ECG Parameters: P Amplitude, R Amplitude, T Amplitude and P Wave Duration.

\begin{tabular}{|c|c|c|c|c|}
\hline Group & P Amplitude (mv) & R Amplitude (mv) & T Amplitude (mv) & P Wave Duration (s) \\
\hline Control & $0.077 \pm 0.003$ & $0.709 \pm 0.005$ & $0.337 \pm 0.002$ & $0.016 \pm 0.001$ \\
\hline HFD+STZ (3 weeks) & $0.097 \pm 0.001^{*}$ & $0.718 \pm 0.004$ & $0.464 \pm 0.0214^{*}$ & $0.029 \pm 0.001^{*}$ \\
\hline
\end{tabular}

Results represent mean \pm SEM of ten animals per group. Results obtained are significantly different from control group $\left({ }^{*} p<0.05\right)$.

R wave amplitude: There was no significant change in $\mathrm{R}$ wave amplitude in HFD+STZ (3 weeks) group rats in comparison to the control rats (Table 2) indicating absence of development of left ventricular hypertrophy.

T wave amplitude: $\mathrm{T}$ amplitude which signifies ventricular repolarisation was found to be significantly higher $\left({ }^{*} \mathrm{p}<0.05\right)$ in HFD+STZ (3 weeks) animals in comparison to the control rats (Table 2) indicating ventricular arrhythmia.

ST segment height: There was significant $\left({ }^{*} p<0.05\right)$ elevation in ST segment height (Figure 2a) in HFD+STZ (3 weeks) group rats in comparison to the control rats.

QRS interval: There was a significant $\left({ }^{*} \mathrm{p}<0.05\right)$ prolongation represents atrial depolarisation and conduction were found in HFD+STZ (3 weeks) group rats in comparison to the control rats (Table 2) suggesting conduction delay between left and right atrium. of QRS (Figure 2b) interval in HFD+STZ (3 weeks) group rats in comparison to the control rats.

RR interval: There was a no significant change in RR (Figure 2c) interval in HFD+STZ (3 weeks) group rats in comparison to the control rats.

QT interval: QT interval which is a measure of time of ventricular repolarisation was found to be significantly higher in HFD+STZ (3 weeks) group rats in comparison to control rats (Figure 2d).

PR interval: Significant $\left({ }^{*} \mathrm{p}<0.05\right)$ increase in PR interval was observed in HFD+STZ ( 3 weeks) group rats in comparison to the control rats (Figure 2e) indicating delay in atrial conduction.

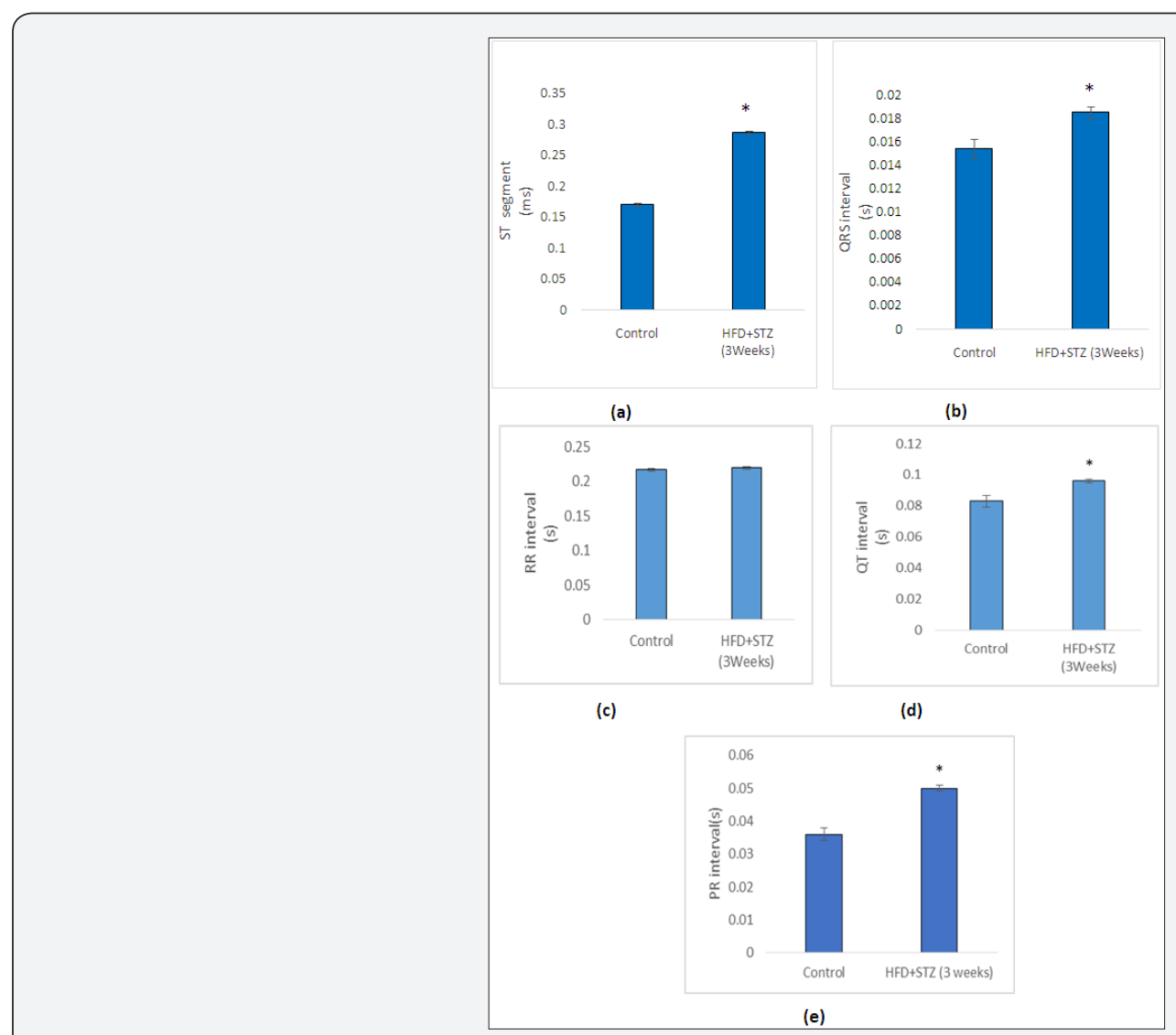

Figure 2: a) ST segment b) QRS interval c) RR interval d) QT interval and e) PR interval. Result expressed as mean \pm SEM of ten animals per group. Results obtained are significantly different from control group $\left({ }^{*} p<0.05\right)$. 


\section{Discussion}

Mortality rate due to CVD in diabetic patient is fifty percent more in comparison nondiabetic individuals [22]. An early detection tool like ECG will help in studying and preventing diabetes induced CVDs [23]. The rat model of T2DM developed by Mansor et al, [20] is an easy, economical and time saving model which can be used to explore multiple pathways which are involved in the development of diabetes induced CVDs [20]. Changes in cardiac metabolism along with hyperglycemia, hyperlipidemia and normoinsulinemia have been reported in the above rat model of T2DM. However, they did not study the ECG changes that may occur in the T2DM rat model and our aim was to fill this lacunae.

The results of the present study demonstrate that the treatment with low dose STZ (25mg/kg; i.p.) and HFD caused hyperglycemia with no change in blood insulin levels (data not shown) validating the development of animal model of diabetes. In the present study, rats administered with HFD+STZ ( 3 weeks) showed a significant increase in the level of CK-MB in serum. HFD+STZ (3 weeks) induced damage in myocardial cell membrane integrity and increased permeability [24]. However, left ventricular hypertrophy was not observed in HFD+STZ (3 weeks) rats indicating the onset of changes in the heart.

HFD+STZ (3 weeks) treated rats showed pathological ECG changes. A significant elevation in $\mathrm{P}$ wave duration and $\mathrm{P}$ amplitude was found. Prolonged $\mathrm{P}$ wave duration and $\mathrm{P}$ amplitude specifically determines the enlargement of the left atrium [25]. Atrial fibrillation occurs due to enlargement of the left atrium which causes delay in conduction between the left and right atria [26-28]. Prolongation of the PR interval may be due to increased atrial conduction time. In addition, a prolonged PR interval results in slow mitral valve closure and diastolic mitral regurgitation [29]. Prolongation of QRS duration in HFD+STZ (3 weeks) rats predicts increase of the cardiovascular risk [30]. The QT interval which was prolonged in HFD+STZ rats, in diabetic patients is a function of progressive worsening of glucose tolerance [31]. ST segment elevation represents HFD+STZ (3 weeks) induced ischemic and non-ischemic zones potential difference and the consequent loss of cell membrane function [32] which is validated by an increase in CK-MB in the present. The R wave amplitude did not change in HFD+STZ rats suggesting that ventricular hypertrophy did not occur which was confirmed by left ventricle weight heart ratio. The ECG changes in the present study indicate that changes in the atrium conduction has taken place in HFD+STZ (3 weeks) rats with some small changes in ventricular conduction. It seems that changes in the cardiac electrical conduction precede ventricular hypertrophy.

\section{Conclusion}

In conclusion, present study demonstrated that HFD and low dose intraperitoneal injection of STZ causes diabetes and that in diabetes cardiac electrical conduction defects set in first before the structural changes appear in the heart. These subtle cardiac electrical changes may be the precipitating factor for the cardiovascular diseases in diabetes

\section{Conflict of interest}

The author(s) declared no conflicts of interest with respect to the authorship and/or publication of this article.

\section{References}

1. Gupta S, Gupta RK, Kulshrestha M, Chaudhary RR (2017) Evaluation of ECG abnormalities in patients with asymptomatic Type 2 Diabetes Mellitus. J Clin Diagn Res 11(4): OC39-0C44.

2. Omanwar S, Gupta C, Dhyani N, Saidullah B (2017). NO (Nitric Oxide) to Type 2 Diabetes Induced Endothelial Dysfunction: Crosstalk with ET-1 (Endothelin-1). Open Acc J of Toxicol 2(1): 555578.

3. Fukui S, Fukumoto Y, Suzuki J, Saji K, Nawata J et al. (2009) Diabetes mellitus accelerates left ventricular diastolic dysfunction through activation of the renin-angiotensin system in hypertensive rats. Hypertens Res 32(6): 472-480.

4. Kannel WB (1983) Prevalence and natural history of electrocardiographic left ventricular hypertrophy. Am J Med 75(3A): 4-11.

5. Seferovic PM, Lalic NM, Seferovic JP, Jotic A, Lalic K, Ristic AD, et al. (2007) Diabetic cardiomyopathy: old disease or new entity? Srp Arh Celok Lek 135(9-10): 576-582.

6. Kannel WB, Levy D, Cupples LA (1987) Left ventricular hypertrophy and risk of cardiac failure: insights from the Framingham Study. J Cardiovasc Pharmacol 10(6): S135-S140.

7. Okin PM, Devereux RB, Lee ET, Galloway JM, Howard BV (2004) Electrocardiographic repolarization complexity and abnormality predict all-cause and cardiovascular mortality in diabetes: the strong heart study. Diabetes 53(2): 434-440.

8. Naas AAO, Davidson NC, Thompson C, Cummings F, Ogston SA, et al (1998) QT and QTc 152 dispersion are accurate predictors of cardiac death in newly diagnosed non-insulin dependent diabetes: Cohort study. Br Med J 316(7133): 745-746.

9. Sawicki PT, Kiwitt S, Bender R, and Berger M (1998) The value of QT interval dispersion for identification of total mortality risk in noninsulin dependent diabetes mellitus. J of Internal Med 243(1): 49-56.

10. Christensen PK, Gall MA, Major-Pedersen A, Sato A, Rossing P, et al. (2000) QTc interval length and QT dispersion as predictors of mortality in patients with noninsulin-dependent diabetes. Scand J Clin Lab Invest 60(4): 323-332.

11. Veglio M, Borra M, Stevens LK, Fuller JH, Perin PC (1999) The relation between QTc interval prolongation and diabetic complications: the EURODIAB IDDM complication study group. Diabetologia 42(1): 6875.

12. Chiu HC, Kovacs A, Blanton RM, Han X, Courtois M, et al. (2005) Transgenic expression of fatty acid transport protein 1 in the heart causes lipotoxic cardiomyopathy. Circ Res 96(2): 225-233.

13. Lindstrom T, Jorfeldt L, Tegler L, Arnqvist HJ (1992) Hypoglycaemia and cardiac arrhythmias in patients with Type 2 diabetes mellitus. Diabetic Med 9(6): 536-541.

14. Molon G, Targher G, Costa A, Bertolini L, Barbieri E, et al. (2006) Measurement of microvolt T-wave alternans, a new arrhythmic risk stratification test, in Type 2 diabetic patients without clinical cardiovascular disease. Diabetic Med 23(2): 207-210.

15. Stern S, Sclarowsky S (2009) The ECG in diabetes mellitus. Circulation 120(16): 1633-1636. 
16. Bugger H, Abel ED (2009) Rodent models of diabetic cardiomyopathy. Dis Model Mech 2(9-10): 454-466.

17. Belke DD, Betuing S, Tuttle MJ, Graveleau C, Young ME, et al. (2002) Insulin signalling coordinately regulates cardiac size, metabolism, and contractile protein isoform expression. J Clin Invest 109(5): 629-639.

18. Semeniuk LM, Kryski AJ, Severson DL (2002) Echocardiographic assessment of cardiac function in diabetic $\mathrm{db} / \mathrm{db}$ and transgenic $\mathrm{db} /$ db-hGLUT4 mice. Am J Physiol Heart Circ Physiol 283(3): H976-982.

19. Trost SU, Belke DD, Bluhm WF, Meyer M, Swanson E. et al. (2002) Overexpression of the sarcoplasmic reticulum $\mathrm{Ca}^{2+}$ ATPase improves myocardial contractility in diabetic cardiomyopathy. Diabetes 51(4): 1166-1171.

20. Mansor LS, Gonzalez ER, Cole MA, Tyler DJ, Beeson JH et al. (2013) Cardiac metabolism in a new rat model of type 2 diabetes using highfat diet with low dose streptozotocin. Cardiovascular Diabetology 12: 136.

21. Hill R, Howard AN, and Gresham GA (1960) The electrocardiographic appearances of myocardial infarction in the rat. British journal of experimental pathology 41: 633-637.

22. Preis SR, Hwang SJ, Coady S, Pencina MJ, D’Agostino RB et al. (2009) Trends in all-cause and cardiovascular disease mortality among women and men with and without diabetes mellitus in the Framingham Heart Study, 1950 to 2005. Circulation 119 (13): 1728-1735.

23. VanHoose L, Sawers Y, Loganathan R, Vacek JL, Bittel LS, et al. (2010) Electrocardiographic changes with the onset of diabetes and the impact of aerobic exercise training in the Zucker Diabetic Fatty (ZDF) rat. Cardiovasc Diabetol 9: 56.

24. Zhou R, Xu Q, Zheng P, Yan L, Zheng J, Dai G (2008) Cardioprotective effect of fluvastatin on Isoproterenol-induced myocardial infarction in rat. European Journal of Pharmacology 586(1-3): 244-250.
25. Munuswamy K, Alpert MA, Martin RH, Whiting RB, Mechlin NJ (1984) Sensitivity and specificity of commonly used electrocardiographic criteria for left atrial enlargement determined by M-mode echocardiography. Am J Cardiol 53(6): 829-832

26. Magnani JW, Gorodeski EZ, Johnson VM, Sullivan LM, Hamburg NM, et al. (2011) P wave duration is associated with cardiovascular and all-cause mortality outcomes: the National health and nutrition examination survey. Heart rhythm 8(1) 93-100.

27. Luna ABD, Platonov P, Cosio FG, Cosio FG, Cygankiewicz I, et al. (2012) Interatrial blocks: A separate entity from left atrial enlargement: a consensus report. J Electrocardiol 45(5): 445-451.

28. Vepsalainen T, Laakso M, Lehto S, Juutilainen A, Airaksinen J, et al. (2014) Prolonged P wave duration predicts stroke mortality among type2 diabetic patients with prevalent non-major macrovascular disease. BMC Cardiovascular Disorders 14(1): 168-174.

29. Schnittger I, Appleton CP, Hatle LK, Popp RL (1988) Diastolic mitral and tricuspid regurgitation by Doppler echocardiography in patients with atrioventricular block: new insight into the mechanism of atrioventricular valve closure. J Am Coll Cardiol 11(1): 83-88.

30. Howarth FC, Jacobson M, Naseer O, Adeghate E (2005a) Long-term effects of streptozotocin-induced diabetes on the electrocardiogram, physical activity and body temperature in rats. Exp Physiol 90(6): 237 245 .

31. Solini A, Passaro A, D’Elia K, Calzoni F, Alberti L, Fellin R (2000) The relationship of plasma glucose and electrocardiographic parameters in elderly women with different degrees of glucose tolerance. Aging (Milano) 12(4): 249-255.

32. Soliman EZ, Prineas RJ, Case LD, Russel G, Rosamond W, et al. (2011) Electrocardiographic and clinical predictors separating atherosclerotic sudden cardiac death from incident coronary heart disease. Heart 97(19): 1597-1601.

\section{Your next submission with Juniper Publishers will reach you the below assets}

- Quality Editorial service

- Swift Peer Review

- Reprints availability

- E-prints Service

- Manuscript Podcast for convenient understanding

- Global attainment for your research

- Manuscript accessibility in different formats

( Pdf, E-pub, Full Text, Audio)

- Unceasing customer service

Track the below URL for one-step submission https://juniperpublishers.com/online-submission.php 